\title{
THE THICK DISC POPULATION OF THE GALAXY
}

\author{
A.C. ROBIN, M. HAYWOOD, D.K. OJHA AND O. BIENAYMÉ \\ Observatoire de Besançon, BP1615, F-25010 Besançoncedex \\ AND \\ M. CRÉZÉ \\ Observatoire de Strasbourg, 11 rue de l'Université, F-67000 \\ Strasbourg
}

The availability of photometric and astrometric star counts on large fields well distributed in the Galaxy allows us to measure the characteristics of the thick disc population. We use a total of 19 data sets in UBV towards a dozen directions in order to measure the scale height, scale length, local density and metallicity of the intermediate population.

The Besançon model of population synthesis (Robin \& Crézé (1986), Bienaymé et al. (1987), Haywood (1994)) is used to simulate catalogues. A grid of models is computed with varying thick disc parameters (scale height, scale length, local density, metallicity) and compared with data sets via a maximum likelihood test.

The derived characteristics of the thick disc show that it is a population distinct from the disc and from the halo. It has a scale height of $780 \mathrm{pc} \pm$ $50 \mathrm{pc}$, a scale length of $2500{ }_{-200}^{+400} \mathrm{pc}$ and a local density of $5.6 \%$ of the disc. Its mean metallicity is $-0.8 \pm 0.1 \mathrm{dex}$. There is no evidence for any vertical metallicity gradient, while a radial gradient may be present (in the range 0 to $-0.15 \mathrm{dex} / \mathrm{kpc}$ ). The implications for the thick disc formation are discussed in Robin et al. (1995, preprint).

\section{References}

Bienaymé, O., Robin, A.C., Crézé, M., 1987, A\&A 180, 94.

Haywood M., 1994, Thèse de doctorat, Observatoire de Paris

Robin, A.C., Crézé, M., 1986, A \& A 157, 71 